\title{
L'INFLUENCE DE L'ALIMENTATION SUR LE DÉVELOPPEMENT DES GONADES ET ANALYSE MORPHOLOGIQUE DE LA MATURATION DU Hyphessobrycon callistus ET DU Trichogaster trichopterus (PISCES, TELEOSTEI)*
}

\author{
MARIA CECÍLIA BERNARDES PADILHA TRIPPIA
}

\author{
Département de Biologie Cellulaire, Université Fédérale du Parana, Caixa Postal 19031, CEP 81504-990 - \\ Curitiba-PR, Brasil.
}

\begin{abstract}
Studies on the Hyphessobrycon callistus and Trichogaster trichopterus (Pisces, Teleostei) gonadal development in regard to nourishment were carried out. The following parameters were studied: the total length, total weight and gonads weight; the gonads indexes; the distribution of the oocytary diameter in oocytes dissociated and the maturity stages of ovaries and testicles. The total length and weight were estimated to be directly proportional to the amount of nourishment offered in both species, as well as the gonads weight of T.trichopterus. In $H$. callistus the largest oocytary diameter was observed in the treatment of the lowest level of nourishment offered, and the largest oocytary diameter of T. trichopterus was observed in the treatment of the highest level of nourishment offered. In T. trichopterus the development oocytary phases and the scales of ovaries and testicles maturity proposed were similar to the ones observed in the literature for other species of fresh water Teleosts. The existence of multiple spawning in both species was estimated. The gonadal maturation was less intense in the treatment of the lowest level of nourishment offered, in T. trichopterus.
\end{abstract}

Key Words: Reproduction, Nourishment, Maturation, Gonads, Teleostei, Characidae, Belontiidae.

RESUMO - Foi realizado estudo do comprimento total, peso total e peso das gônadas; dos índices gonadais; da distribuição do diâmetro ovocitário em ovócitos dissociados, assim como de seus maiores diâmetros por exemplar; e dos estádios de maturidade encontrados nos ovários e testículos, em relação à quantidade de alimento ofertado em três diferentes tratamentos de cada experimento, com Hyphessobrycon callistus e com Trichogaster trichopterus (Peixes, Teleostei). O comprimento total e o peso revelaram a tendência de serem diretamente proporcionais à quantidade de alimento ofertado em ambas as espécies, assim como o peso das gônadas em $T$. trichopterus. Em $H$. callistus o maior diâmetro ovocitário foi observado no tratamento de menor oferta alimentar, sendo que o maior diâmetro ovocitário de T. trichopterus refere-se ao tratamento de maior oferta alimentar. Em T. trichopterus as fases de desenvolvimento ovocitário encontradas e as escalas de maturidade de ovários e testículos propostas, foram semelhantes às observadas na literatura para outras espécies de Teleósteos dulceaqüícolas. Foi estimada a existência de desova múltipla em ambas as espécies. A maturação gonadal foi menos intensa no tratamento de menor oferta alimentar de T. trichopterus.

Palavras-Chave: Reprodução, Alimentação, Maturação, Gônadas, Teleostei, Characidae, Belontiidae.

\section{Introduction}

Chez tous les êtres vivants, la reproduction a de caractéristiques biologiques qui résultent, d'une part, des potentialités propres à chaque espèce et, d'autre part, des exigences imposées par l'environnement. On sait que les styles reproductifs des espèces qui vivent dans des milieux écologiquement instables rassemblent des tactiques comme la fécondité élevée, avec des ovocytes très nombreux et de petite taille, la durée de maturation sexuelle moins longue, la ponte par période restreinte de temps, la longévité moins longue, une plus grande mortalité des adultes, l'absence de

*Extrait de la thèse de "Master of Science" présentée à l’Université Fédérale du Parana-Brésil, le 04-7-1995.

${ }^{1}$ Ex-Boursière de la Fondation Coordination de Perfectionnement de Personnel à Niveau Supérieur (CAPES). Adresse actuelle: Caixa Postal 2806, CEP 80001-970, CuritibaPr, Brasil. soins à la progéniture et un grand effort reproductif. En plus, la taille de ces populations est contrôlée par de facteurs indépendants de la densité de la population. Les espèces que vivent dans des milieux stables, à leur tour, ont l'habitude de présenter une basse fécondité, avec des ovocytes plus grands et peu nombreux, une plus grande durée de maturation sexuelle, la ponte par période prolongée pendant l'année, de forme plus au moins continue, une plus grande longévité, une plus petite mortalité des adultes, des soins à la progéniture et un plus petit effort reproductif. La taille des populations qui vivent dans des milieux écologiquement stables, est contrôlée par des facteurs dépendants de la densité de la population (MARGALEF, 1977).

Leurs réponses reproductives à ce niveau sont connues, le même ne se passe pas avec exactitude en ce qui concerne les variations cytologiques et histologiques qui touchent la maturation des 
gonades. De quelle façon le procès de gamétogénèse s'ajuste aux caractéristiques de l'environnement? Quels sont les effets qu'une plus grande quantité de nourriture proposée pourrait causer au développement ovocytaire et testiculaire? C'est possible que la taille des ovocytes augmente, ayant une diminution numérique, avec la finalité de maximiser le nombre de survivants? Ces questions et d'autres ont conduit à ce travail, chez deux différents espèces de Teleostei. Voulant, ainsi, identifier les aspects cytologiques de la maturation ovocytaire du Hyphessobrycon callistus (famille Characidae) et du Trichogaster trichopterus (famille Belontiidae) et analyser de quelle façon les développements ovocytaire et spermatique répondent aux variations de la quantité de nourriture proposée, chez ces espèces.

\section{Méthodologie}

Soixante-quinze exemplaires du Hyphessobrycon callistus, (Boulenger, 1900) et 75 exemplaires du Trichogaster trichopterus, Pallas, 1770, immatures et avec le même âge, ont été acquis. Ces spécimens n'ont subi aucun traitement hormonal. Les 75 exemplaires de chaque espèce ont été distribués de façon aléatoire dans trois traitements: 6 aquariums identiques de 451 , avec 25 exemplaires chacun. La seule variante existente entre les traitements de chaque expérimentation a été la quantité de nourriture (ration) proposée aux animaux: $0,20 \mathrm{~g}$ au traitement $1 ; 0,40 \mathrm{~g}$ au traitement 2 et $0,60 \mathrm{~g}$ au traitement 3 , à chaque dose. Les exemplaires ont été nourris simultanément, trois fois par jour (le matin, à midi et l'après-midi) du lundi au vendredi et deux fois par jour (le matin et l'après-midi) le samedi et le dimanche. Le $\mathrm{pH}$ de l'eau s'est maintenu constant entre les traitements, étant ainsi, la quantité de nourriture proposée la seule variante entre les aquariums de chaque expérimentation. La photopériode a coïncidée avec celle observée dans la nature, pour tous les traitements. La ration utilisée dans les expérimentations s'appelle ALCONKOI et a comme composition essentielle: de la farine de poisson, du son de soja, de la farine de maïs, de la farine d'avoine, de la farine de feuilles d'orge, de la graisse animale, du sel et des jaunes d'oeufs. Le sacrifice simultané de tous les exemplaires s'est passé juste après l'observation du début du comportement reproductif, soit avant la première ponte.

On a réalisé l'étude de la longueur totale, du poids total et du poids des gonades. Les poids inférieurs à un dizième de gramme n'ont pas été considérés. Les valeurs de l'indice gonadosomatique (IGS) et de l'indice gonadal (IG) ont été calculés individuellement et puis groupés par traitement, pour chaque sexe. Les moyennes tantôt d'IGS comme d'IG ont été liées aux traitements de chaque expérimentation. Une portion de chaque paire d'ovaire, déjà fixée en formol à $10 \%$ a été transférée à l'alcool à $70^{\circ} \mathrm{GL}$, dissociée attentivement avec l'aide de pinces et d'aiguilles et destituée de tous les résidus de tissu. Il a été collecté un échantillon au hasard de la masse totale d'ovocytes dissociés ou peu groupés, en suspension dans l'alcool à $70^{\circ} \mathrm{GL}$, avec une pipette Pasteur, ayant le soin d' homogénéiser très bien la suspension au moment de la collecte. Le diamètre de chaque ovocyte a été mesuré au microscope de lumière, avec l'aide d'une oculaire micrométrique. On a estimé comme représentatif de chaque unité la valeur de son diamètre horizontal moyen. Il a été calculé la fréquence relative d'ovocytes par classe de diamètre. La distribution de fréquences des diamètres des ovocytes des trois traitements, concernant les gonades dans un même stade de développement, a été groupée, et la fréquence moyenne d'ovocytes par classe de diamètre calculée. Il a été réalisé la comparaison entre les dix plus grands diamètres ovocytaires et les traitements.

Les gonades fixées en formol à $10 \%$ ont été incluses en paraffine selon la technique de routine. Les coupures avec une épaisseur moyenne de $6 \mu \mathrm{m}$, ont été colorées avec Hematoxiline-Eosine et ont été observées au microscope de lumière. Les phases de développement ovocytaire et les stades de maturation gonadal trouvées ont été décrites.

\section{Résultats}

Phases de développement ovocytaire. Selon les observations microscopiques il a été possible de distinguer cinq phases différentes de développement ovocytaire.

PHASE I (Cellules germinatives jeunes). Ces cellules, les plus petites, avec un diamètre moyen de $27,2 \mu \mathrm{m}$, ont la forme sphérique et sont fréquemment trouvées en groupes, formant des nids insérés dans les lamelles, généralement dans les régions de bonne irrigation sangüine. Elles ont le cytoplasme très réduit, avec peu de basophilie et de nucléoles arrondis et basophiles, ayant bonne affinité teintée avec l'hematoxiline (Fig. 1A1). Ces cellules ont été trouvées chez le T. trichopterus. Les ovaires du $H$. callistus ont appartenu seulement au stade mûr, ce qui a difficulté la visualisation des ovocytes dans cette phase.

PHASE II (Ovocytes basophiles du stock de réserve). Ayant atteint cette phase les ovocytes maintiennent la forme sphérique, mais leur volume général augmente beaucoup. Le diamètre moyen 
chez le $H$. callistus est de $60 \mu \mathrm{m}$ et de $66,45 \mu \mathrm{m}$ en ce qui concerne l'espèce $T$. trichopterus. C'est possible d'observer la séparation des groupements en nids, peut-être à cause de l'augmentation du volume général, et le cytoplasme devient bien défini et plus basophile. Le noyau se maintien sphérique et central, les nucléoles sont plus nombreux, petits et basophiles, et ont la tendance à migrer vers la périphérie du noyau. Au fur et à mesure que les cellules augmentent de volume, le noyau se présente avec une plus grande quantité de nucléoles (Fig. 1A2 et 1B1).

PHASE III. Les ovocytes sont plus grands, de forme sphérique ou ovoïde, ayant un diamètre moyen de $127,5 \mu \mathrm{m}$ chez le $H$. callistus et de $120,45 \mu \mathrm{m}$ chez le $T$. trichopterus.. Le noyau, aussi plus grand, ne croît pas à la même proportion que le cytoplasme et se montre avec une plus petite affinité par l'hematoxiline, étant son contour irrégulier dans quelques cas. Les nucléoles sont plus nombreux et plus petits que dans la phase précédente, se maintient à la périphérie. Il est possible d'observer dans cette phase, le début de la déposition de substances de réserve dans le cytoplasme, moins basophile chez le H. callistus, ainsi que sa croissance accélerée. Ces vésicules sont trouvées au cytoplasme périphérique, avec une tendance à augmenter beaucoup en nombre et en taille et à occuper presque la totalité du cytoplasme (Fig. 1A2 et 1B1).

PHASE IV. Cette phase est caractérisée par le début de l'apparition, au cytoplasme, de quelques petits granules acidophiles, de coloration rosâtre intense, entre les vésicules. Ils sont formés premièrement, dans la périphérie du cytoplasme, étant, au début, très petits et ensuite augmentant de volume. Les ovocytes augmentent beaucoup de volume: $267,27 \mu \mathrm{m}$ et $197,55 \mu \mathrm{m}$, en moyenne, chez les $H$. callistus et $T$. trichopterus, respectivement. Avec la forme sphérique, ovoïde ou irrégulière, à cause de la pression entre les cellules voisines, son cytoplasme continue a croître se maintenant basophile, fortement coloré par l'hematoxiline. Le noyau acidophile présente un contour sinueux et les nombreux nucléoles sont périphériques (Fig. 1A3 et 1B2).

PHASE V. La forme des ovocytes est semblable à laquelle de la phase précédente, mais ses diamètres ont augmenté considérablement: $472,5 \mu \mathrm{m}$ en moyenne chez le $H$. callistus et $411,45 \mu \mathrm{m}$ en moyenne chez le $T$. trichopterus. Cela est occasionné par la grande augmentation du nombre de vitellus acidophile. Le noyau a maintenu ses caractéristiques précédentes, avec un contour irrégulier, formant quelques petites invaginations où généralement les nucléoles sont logés. $\mathrm{La}$ basophilie cytoplasmatique a disparu presque totalement chez le $T$. trichopterus (Fig. 1A4 et 1B3).

$\mathrm{Au}$ niveau de l'analyse procédée, en ce qui concerne les cellules germinatives jeunes il n'a pas été observé aucun enveloppe cellulaire, même en étant déjà entourées par de cellules somatiques, futures cellules de la thèque et folliculaires. Les ovocytes basophiles du stock de réserve sont entourés par un épithélium du type folliculaire simple pavementé, ainsi comme les ovocytes dans la phase III (Fig. 2A1, 2A2 et 2B1). Chez les ovocytes dans la phase III la membrane vitelline est déjà formée (Fig. 2A2 et 2B1). Dans les phases IV et $\mathrm{V}$ les cellules folliculaires se maintiennent dans une seule couche pavementée (Fig. 2A3, 2A4, 2B1 et $2 \mathrm{~B} 2$ ), et la membrane vitelline des ovocytes dans la phase $\mathrm{V}$ est devenue plus large (Fig. 2A4 et 2B2).

Caractérisation des stades trouvés de maturation des ovaires. A travers la littérature et la comparaison entre les observations microscopiques, une échelle de maturation ovarienne qui comprend cinq stades différents a été élaborée.

STADE A - Imature ou vierge. Seulement les individus qui n'ont pas ancore débuté sa première maturation sexuelle appartient à ce stade. Les ovaires immatures sont caractérisés par l'organisation et par la grande quantité de cellules. Ceux-là se trouvent dans les phases I et II du développement ovocytaire, étant très rare la présence d'ovocytes dans la phase III. Les nids de cellules germinatives (phase I) se localisent généralement à la proximité des petits vaisseaux sanguins (Fig. 3A1).

STADE B1 - En maturation initiale. Ce estade englobe, en plus des ovocytes dans les phases I et II présents dans le stade précédent, les ovocytes de la phase III. Les ovocytes dans la phase IV sont trouvés très rarement (Fig. 3A2).

STADE B2 - En maturation intermédiare. Font partie de ce stade, les ovaires avec des ovocytes dans les phases I, II, III, IV et occasionnellement, des ovocytes dans la phase V (Fig. 3A3).

STADE B3 - En maturation avancée. L'analyse des gonades nous montre une grande variété de cellules, que caractérise son identification. Nous trouvons des ovocytes dans les phases II, III, IV et V. D'autre part, ovocytes dans la phase I n'ont pas été observés (Fig. 3A4). 
STADE C - Mûr. Font partie de ce stade les ovaires avec des ovocytes dans les phases II, III, IV et un grand nombre d'ovocytes dans la phase V, occupant presque toute l'aire de la coupure. Les ovocytes dans la phase I n'ont pas été trouvés (Fig. $3 \mathrm{~A} 5$ et 3B1). Les ovaires dans les stades A, B1, B2 et $\mathrm{B} 3$ n'ont pas été trouvés chez l'espèce $H$. Callistus.

Fig. 1. Ovocyte dans la phase I (I) (1A1), ovocytes dans la phase II (II) (1A2 et 1B1), ovocytes dans la phase III (III) (1A1 et 1B1), ovocytes dans la phase IV (IV) (1A3 et 1B2) et ovocytes dans la phase V (V) (1A4 et 1B3) du Trichogaster trichopterus (A) et du Hyphessobrycon callistus (B). Noyaux (N) nucléoles (petites fléches), vesicules (fléches transparentes) 0et granules acidophiles (fléches courbes). 
L’influence de l'alimentation sur le Développement des Gonades des Poissons

Fig. 2. Vue partiale d'ovocytes dans la phase II (II) (2A1 et 2B1), d'ovocytes dans la phase III (III) (2A2 et 2B2 ), d'ovocytes dans la phase IV (IV) (2A3 et 2B2) et d'ovocytes dans la phase V (V) (2A4 et 2B3) du Trichogaster trichopterus (A) et du Hyphessobrycon callistus (B). Membrane viteline (M V) et cellules folliculaires (fléches).

Caractérisation des stades trouvés de maturation des testicules. Selon les observations microscopiques, et les comparaisons avec les informations disponibles pour d'autres espèces de Teleostei, les testicules du $H$. callistus et du T. trichopterus de cet essai ont été caractérisés comme appartenant aux stades suivants de maturation gonadal: stade IV ou en maturation avancée et stade $\mathrm{V}$ ou mûr.

Dans le stade IV ou en maturation avancée, la lumière $\mathrm{du}$ tube seminiphèré s'est présentée presque totalement remplie par de spermatozoïdes (Fig. 4A1 et 4B1) et totalement remplie dans le estade $\mathrm{V}$ ou mûr, étant encore observé la présence de kistes contenant de cellules spermatogeniques (Fig. 4A2 et 4B2).

Tantôt dans les ovaires tantôt dans les testicules il n'a pas été trouvé des indices élevés de corps atrétiques.

Analyse des stades de maturation des ovaires et des testicules par rapport aux traitements. Les gonades ont été caractérisées en 5 stades: immatures, en maturation initiale, en maturation intermédiaire, en maturation avancée et mûr. Chez le $H$. callistus ont été trouvés seulement deux stades de maturation sexuelle: en maturation et mûr. De cette façon, pour les traitements 1,2 et 3 de l'espèce $T$. trichopterus, ont été visualisées, respectivement: 3 , zéro et zéro gonades immatures; 5,2 et 1 en maturation initiale; 3,7 et 6 en maturation intermédiare; zéro, 5 et 4 en maturation avancée; et 2 , 6 et 3 gonades mûres (Fig. 5B). Pour les traitements 1,2 et $3 \mathrm{du} H$. callistus ont été observées, respectivement, zéro, 5 et 1 gonades en maturation et 2, 5 et 5 gonades mûres (Fig. $5 \mathrm{~A}$ ).

L'analyse des proportions a démontré l'existence de différence significative entre les traitements 1 et 2 , et 1 et 3 chez le $T$. trichopterus, n'ayant pas de différence entre les traitements 2 et 3 . Pour obtenir ces résultats a été nécessaire de grouper les stades immature et en maturation initiale, et en maturation avancée et mûr.

Selon le test de comparaisons multiples, toutes les informations concernant l'espèce $H$. callistus n'ont pas présenté de différences significatives entre les traitements. 
Fig. 3. Ovaire imature (3A1), ovaire en maturation initiale (3A2), ovaire en maturation intermédiaire (3A3), ovaire en maturation avancée (3A4) et ovaires mûrs (3A5 et 3B1) du Trichogaster trichopterus (A) et du Hyphessobrycon callistus (B). Ovocytes dans la phase II (II), ovocytes dans la phase III (III), ovocytes dans la phase IV (IV) et ovocytes dans la phase V (V). $140 \mathrm{X}$.

Longueur totale, poids total et poids des gonades. Chez les mâles et chez les femelles du H. callistus, la moyenne de la longueur totale et la moyenne du poids total ont augmenté progressivement jusqu'au traitement proposant la plus grande quantitée de nourriture. La plus grande moyenne du poids des gonades a été observé au traitement 3 et la plus petite au traitement 2 (Tableau 1).

Les plus grandes moyennes par rapport au $T$. trichopterus, ont été observées au traitement proposant la plus grande quantité de nourriture et les plus petites moyennes au traitement proposant la plus petite quantité de nourriture (Tableau 2). 
L'influence de l'alimentation sur le Développement des Gonades des Poissons

Tableau 1. Moyennes de la longueur totale (LT), poids total (PT) et poids des gonades (PG) par traitements des deux sexes du Hyphessobrycon callistus. $\mathrm{N}=$ nombre d'exemplaires.

\begin{tabular}{c|cc|cc|ccc}
\hline Traitements & $\mathrm{N}$ & $\mathrm{LT}(\mathrm{mm})$ & $\mathrm{N}$ & $\mathrm{PT}(\mathrm{g})$ & $\mathrm{N}$ & $\overline{\mathrm{PG}}(\mathrm{g})$ \\
\hline 1 & 25 & 32,56 & 25 & 0,39 & 5 & 0,04 \\
\hline 2 & 25 & 33,17 & 25 & 0,43 & 6 & 0,02 \\
\hline 3 & 24 & 33,90 & 24 & 0,45 & 4 & 0,05 \\
\hline
\end{tabular}

Tableau 2. Moyennes de la longueur totale (L), poids total (PT) et poids des gonades (PG) par traitements des deux sexes du Trichogaster trichopterus. $\mathrm{N}=$ nombre d'exemplaires.

\begin{tabular}{c|cc|cc|cc}
\hline Traitements & $\mathrm{N}$ & $\overline{\mathrm{LT}}(\mathrm{mm})$ & $\mathrm{N}$ & $\overline{\mathrm{PT}}(\mathrm{g})$ & $\mathrm{N}$ & $\overline{\mathrm{PG}}(\mathrm{g})$ \\
\hline 1 & 25 & 63,52 & 25 & 2,58 & 16 & 0,05 \\
\hline 2 & 25 & 65,44 & 25 & 3,17 & 16 & 0,07 \\
\hline 3 & 24 & 68,96 & 23 & 3,85 & 14 & 0,10 \\
\hline
\end{tabular}

Fig. 4. Testicules en maturation avancée (4 A 1 et 4B1) et testicules mûrs (4A2 et 4B2) du Trichogaster trichopterus (A) et Hyphessobrycon callistus (B). Spermatozoïdes (Spz) dans des tubes seminiphères et d'autres cellules spermatogeniques (Spg). 140 X. 
Fig. 5. Fréquence des stades de maturation des ovaires et des testicules du Hyphessobrycon callistus (A) et du Trichogaster trichopterus $(\mathrm{B})$, en relation aux 3 traitements $(\mathrm{f}(\%))=$ fréquence en pourcentage; Trait. = traitements.

Selon l'analyse de variance et le test de Tukey, avec 5\% de signification, seulement entre les données du poids des gonades du $\mathrm{H}$. callistus par rapport au traitement 2 et 3, il y a eu une différence significative. Il n'y a pas observé de différence significative par rapport au poids des gonades du T. trichopterus, entre les traitements. La moyenne de la longueur totale et du poids total du traitement 3 de cette espèce, a été significativement supérieur aux autres traitements.
Chez les femelles du H. callistus il a été observé une tendance à la correlation negative entre le poids total et les traitements, ayant la plus grande valeur du poids total au traitement 1 et la plus petite au traitement 2 (Fig. 6A2). La plus grande valeur de la longueur totale, chez les mâles, a été observée au traitement 1 et la plus petite valeur au traitement 2 . Chez ces exemplaires la plus grande valeur du poids total a été enregistrée au traitement 2 et la plus petite valeur au traitement 1 (Fig. 6A1). 
Fig. 6. Relation entre la longueur totale (L T) et les traitements, et le poids total (PT) et les traitements, pour exemplaires mâles (1) et femelles (2) du Hyphessobrycon callistus (A) et du Trichogaster trichopterus (B). Trait. = traitements.

Chez les femelles et chez les mâles de l'espèce T. trichopterus, analysés séparément, a été enregistré une tendance à la corrélation positive entre longueur totale, poids total et traitements ( $\mathrm{P}$ $>0,05$ ) (Fig. 6B1 et 6B2).

Les proportions de gonades immensurables par rapport aux traitements 1,2 et 3 sont respectivement de $84,38 \%, 80 \%$ et $86,21 \%$ chez le H. callistus et de $36 \%, 36 \%$ et $39,13 \%$ chez le $T$. trichopterus. Ainsi, il n'a pas été possible de faire des calculs statistiques en ce qui concerne les analyses réalisées séparément pour chaque sexe.

Indices de gonades. Chez les mâles du H. callistus, a été observé que tantôt l'IG comme l'IGS sont plus grands au traitement proposant la plus petite quantité de nourriture (Fig. 7 - 1A1 et 7 - 2A1). Le plus grandes valeurs d'IG et d'IGS chez les femelles de cette même espèce, ont été enregistrées au traitement 3 et les plus petites valeurs au traitement 1 et 2, respectivement (Fig. 7 - 1A2 et 7 2A2).

En ce qui concerne l'espèce $T$. trichopterus, l'indice gonadal (IG) et l'indice gonado-somatique
(IGS) des femelles ont présenté une tendance à la correlation positive avec la quantité de nourriture proposée $(\mathrm{P}>0,05)$ (Fig. $7-1 \mathrm{~B} 2$ et 7 - 2B2). D'autre part, chez les mâles, la plus grande valeur d'IGS correspond au traitement 2 et la plus petite valeur au traitement $1(\mathrm{P}>0,05)$ (Fig. 7 - 2B1). Dans celui-là, l' IG a eu la tendance d'être plus grand au traitement $3(\mathrm{P}>0,05)$ (Fig. 7 - 1B1).

Il n'a pas été possible de faire de calculs statistiques, à cause du grand pourcentage de gonades avec un poids inférieur à un dizième de gramme.

Distribution du diamètre ovocytaire. Les ovaires du $H$. callistus appartenant au traitement 1 ont enregistré les ovocytes avec le plus grand diamètre, mesurant jusqu'à $2130 \mu \mathrm{m}$. Le traitement 2 a enregistré le diamètre maximum de $390 \mu \mathrm{m}$ et le traitement 3 le diamètre maximum de $480 \mu \mathrm{m}$ (Fig. 8A).

Les plus grands diamètres ovocytaires chez le $T$. trichopterus ont été observés aux traitements 3,2 et 1, respectivement: $390 \mu \mathrm{m}, 360 \mu \mathrm{m}$ et $180 \mu \mathrm{m}$ (Fig. $8 \mathrm{~B})$. 
Fig. 7. Relation entre l'indice gonadal (I G) (1) et les traitements et entre l'indice gonado somatique (IGS) (2) et les traitements, pour exemplaires mâles (1) et femelles (2) du Hyphessobrycon callistus (A) et du Trichogaster trichopterus (B). Trait. $=$ traitements. 
L'influence de l'alimentation sur le Développement des Gonades des Poissons

Fig. 8. Moyennes des diamètres ovocytaires par référence aux 3 traitements du Hyphessobrycon callistus (A) et du Trichogaster trichopterus $(\mathrm{B}) . \mathrm{f}(\%)=$ fréquence en pourcentage; $\mathrm{D}=$ diamètre; (nombre d'exemplaires/ quantité probable d'ovocytes mesurés par exemplaires $=1200$ ).

Les 10 diamètres ovocytaires les plus importants. Parmi tous les ovaires analysés les plus grandes et les plus petites moyennes observées dans l'espèce H. callistus correspondent aux traitements 1 et 2 , respectivement (Tableau 3). Chez le T. trichopterus la plus grande moyenne a été constatée au traitement 2 et la plus petite moyenne au traitement
1 (Tableau 3). Aucune valeur, en ce qui concerne l'espèce $H$. callistus, a été significative. Seulement la différence des valeurs moyennes entre les traitements 2 et 3 du $T$. trichopterus, a été significative. Ces résultats statistiques ont été obtenus à travers l'analyse de variance et le test de Tukey, avec 5\% de signification.

Tableau 3. Moyennes des 10 plus grands diamètres ovocytaires du Hyphessobrycon callistus et du Trichogaster trichopterus (en $\mu \mathrm{m}) . \mathrm{N}=$ nombre d'exemplaires.

\begin{tabular}{c|cc|cc}
\hline Traitements & $\mathrm{N}$ & H. callistus & $\mathrm{N}$ & T. trichopterus \\
\hline 1 & 6 & 1208 & 6 & 307 \\
\hline 2 & 4 & 698 & 13 & 480 \\
\hline 3 & 3 & 813 & 11 & 464 \\
\hline
\end{tabular}




\section{Discussion}

Dans ce travail, selon les observations microscopiques il a été possible de distinguer cinq phases différentes de développement ovocytaire en ce qui concerne chaque espèce. Mais, chez les ovocytes de la sardine ont été classifiés en trois phases par WORSMANN et MANDELLI (1976). Alors, le nombre de phases de développement ovocytaire peut varier selon les espèces, à cause de leur propres caractéristiques, mais aussi à cause de l'auteur, en ce qui concerne les méthodes par lui utilisées.

Contrairement à ce qui a été observé chez le $T$. trichopterus, les ovocytes du Oreochromis aureus appartenant à la phase I (cellules germinatives jeunes) ont la forme d'un polyèdre et non la forme sphérique (GARCIA et PHILLIP, 1986). Dans les ovocytes du $H$. callistus appartenant à la phase II, on peut suggérer que l'intensité de la basophilie cytoplasmatique indique une importante activité de synthèse de protéines (WORSMANN et MANDELLI, 1976 apud ISAAC-NAHUM et al., 1983). Et que la dynamique des nucléoles pendant les étapes du développement ovocytaire suggèrent l'important rôle du noyau pendant la prèvitellogenèse (HOUILLON, 1972).

La présence de vésicules de lipides dans la périphérie du cytoplasme, caractérise les ovocytes de la phase III chez le Brycon cephalus (ZANIBONI FILHO et RESENDE, 1988) semblable à ce qui a été observé par ISSACNAHUM et al. (1983) chez le Sardinella brasiliensis et par CHAVES et VAZZOLER (1984 apud ZANIBONI FILHO et RESENDE, 1988) chez le genre Semaprochilodus. D'autre part, selon NAGAHAMA (1983 apud ZANIBONI FILHO et RESENDE, 1988) en général, la déposition de vésicules lipidiques commence au cytoplasme périnucléaire. Chez le $H$. callistus et chez le $T$. trichopterus les vésicules sont trouvés au cytoplasme périphérique et on a pu observer que dans la phase V leurs volumes augmentent. Cela peut être compris comme une adaptation à la flutuation de l'oeuf, parce que le cytoplasme en portant beaucoup de vésicules peut-être moins dense que l'eau. Selon CRAIK et HARVEY (1987 apud CHAVES, 1989), la baisse densité des oeufs est garantie surtout par sa teneur en lipides.

Les ovaires chez le $T$. trichopterus et chez le $H$. callistus ont été caractérisés en cinq stades différents, à travers la littérature et la comparaison entre les observations microscopiques. Sont-ils: STADE A, immature ou vierge; STADE B1 ou en maturation initiale; STADE B2 ou en maturation intermédiaire; STADE B3 ou en maturation avancée; et STADE $\mathrm{C}$ ou mûr. Chez le $T$. trichopterus tantôt dans les stades B3 comme dans le stade $\mathrm{C}$ on a trouvé des ovocytes dans les phases II, III, IV et V, mais cela a été dans le stade C qu'il y a eu la plus grande quantité d'ovocytes dans la phase V. D'autre part, chez le H. callistus, n'ont été trouvé que des ovaires dans le STADE C ou mûr, avec des ovocytes dans les phases II, III, IV et un grand nombre d'ovocytes dans la phase V. Ainsi, à cause de l'existence de plus de deux lots vitellogeniques en ovaires de $T$. trichopterus, que on a pu supposer l'existence de frais multiple. CHAVES (1985 apud ROMAGOSA et al., 1988) a suggéré l'existence de deux possibilités: "frais unique" et "frais multiple". Selon l'auteur, la première correspond à seulement 2 lots d'ovocytes, en sachant que le lot d'ovocytes avitellogeniques doit se maintenir après l'élimination du lot vitellogenique. Dans le frais multiple, il existe plus d'un lot vitellogenique, dérivant quelques frais. Mais cela ne peut pas toujours correspondre à la réalité.

Beaucoups de groupes vitellogeniques ont été trouvés aussi chez le Prochilodus scrofa, mais au moment exact de la ponte, cette espèce a présenté seulement 2 lots ovocytaires, un d'ovocytes avitellogeniques et un autre d'ovocytes vitellogeniques (FENERICH-VERANI et al., 1984 et ROMAGOSA et al., 1985 apud ROMAGOSA et al., 1988). Ce comportement a été observé aussi chez Merluccius gayi peruanus, étant dénommé d'ovogenèse continue cumulative (ADRIANOV et LISOVENKO, 1983 apud ROMAGOSA et al., 1988)

Selon la littérature et les observations microscopiques, les testicules du $H$. callistus et du T. trichopterus ont été classés comme appartenant aux stades: en maturation avancée et mûr. LEHRI (1967 apud CECILIO et AGOSTINHO, 1991) a dénoté une échelle avec cinq stades pour les testicules du Clarias batrachus. BARBIERI (1981 apud CECILIO er AGOSTINHO, 1991) a determiné trois stades de maturation testiculaire chez le Gymnotus carapo. MATSUURA et al., (1987) a classifié quatre stades de maturation testiculaire chez le Pagrus major.ZANIBONI FILHO et RESENDE (1988) ont observé chez Brycon cephalus cinq stades de maturation testiculaire. Mais, les plus grands nombres de stades ont été déterminés par LASIAK (1983 apud CECILIO et AGOSTINHO, 1991), sept stades chez Liza richardsoni, et par SREENIVASANI (1978 apud CECILIO et AGOSTINHO, 1991), huit stades chez Megalaspis cordyla.

Chez le $T$. trichopterus, les phases de développement ovocytaire rencontrées et les échelles de maturation des ovaires et des testicules proposées ont été semblables à celles observées 
dans la littérature pour d'autres espèces de Teleostei d'eau douce.

En ce qui concerne l'influence de l'alimentation à la longueur totale, poids total et poids des gonades, les résultats obtenus dans ce travail semblent suivre ceux d'ALLEN (1935) qui a conclu que l'augmentation de la quantité de nourriture proposée élève la croissance du Perca fluviatilis.

Pour essayer de comprendre le résultat de la valeur maximale d'IGS c'est intéressant d'observer que les femelles du Protopterus aethiopicus ont de valeurs d'IGS que varient directement avec la maturation des ovocytes et avec le poids des ovaires, mais que les mâles ne démontrent pas d'augmentation de leur IGS après le stade III de maturation gonadal. Cela veut dire que les testicules de cette espèce augmentent en poids seulement jusqu'au stade III (MOSILLE et MAINOYA, 1988). Toutefois les valeurs maximales d'IG et d'IGS chez le $T$. trichopterus n'appartiennent pas au même traitement. Et selon RIMMER (1985) le taux de développement ovocytaire varie significativement entre les exemplaires. De cette façon, c'est possible de suggérer que la petite quantité d'informations associées à cette variation entre les exemplaires, a changé ou compromis les résultats, les rendant douteux.

Les résultats par référence à la moyenne des dix diamètres ovocytaires les plus importants, montrent que chez le $H$. callistus, le plus grand diamètre ovocytaire est au traitement 1, suivi par les traitements 3 et 2 . Le diamètre ovocytaire maximum du traitement 1 a été de $2.130 \mu \mathrm{m}$; ce qui a excédé les expectatives; pas seulement à cause du fait d'appartenir au traitement qui a proposé la plus petite quantité de nourriture, comme d'être extrêmement élevé par rapport aux autres diamètres. Chez le $T$. trichopterus ces résultats se montrent aussi contraires à l'hypothèse que les exemplaires mieux nourris ont tendance à produire des ovocytes avec un plus grand diamètre et ainsi avec une plus grande quantité de vitellus. D'autre part, ce n'est pas très claire si la taille des ovocytes est déjà génétiquement déterminée ou si elle dépend des conditions alimentaires de femelles avant la reproduction (GUISANDE et GLIWICZ, 1992).

Selon l'analyse des stades groupés de maturation des ovaires et des testicules, il n'a pas de différence significative entre les traitements 2 et $3 \mathrm{du} T$. trichopterus; ainsi comme il n'a pas été observé de différence significative entre les traitements du $H$. callistus, en ce qui concerne les stades de maturation des ovaires et des testicules, et l'analyse des dix diamètres ovocytaires les plus importants.

C'est possible alors que la quantité de nourriture proposée au traitement 3 ait été excédante, et que la quantité de nourriture proposée au traitement 2 était suffisante pour nourrir les exemplaires du traitement 3. En sachant que n'ont pas existé de restes de nourriture aux aquariums, on peut imaginer que la qualité de l'eau est devenue faible à cause d'une augmentation des excréments des poissons. C'est important de souligner que la nourriture n'a pas été introduite, selon les proportions établies, chez chaque exemplaire mais librement dans les aquariums. Ce fait a pu causer le meilleur résultat alimentaire des exemplaires génétiquement plus aptes appartenant au même traitement.

Deux mécanismes ont été proposés pour expliquer l'hypothèse que la catégorie nutritive peut altérer la syncronie des evénements gametogéniques. Le premier fait référence au taux d'assimilation de nutriments, et le deuxième au probable niveau critique d'accumulation de nutriments. Ce dernier doit être obtenu avant le début de la gametogenèse (ZIMMERMAN, 1988 apud RUIZ et al., 1992). Chez les femelles adultes du Pleuronectes platessa, la moitié de la croissance des ovaires est observée pendant la période de déficience alimentaire. Ce sont les réserves énergétiques d'autres régions du corps qui doivent suppléer la déficience alimentaire des ovaires, en causant le retard de la croissance ovarienne (DAWSON et GRIMM, 1980). L'excédant d'énergie nécessaire pendant la croissance des gonades, maturation et frais est supprimé par le stock de réserve du corps, dénotant quelques modifications dans la composition biochimique des muscles (DAMBERGS, 1964 et LOVE, 1970 apud MUKHOPADHYAY et SINHA, 1986), ou foie, muscles et gonades de poissons sexuellement mûrs (SHEVCHENKO, 1972; OCHIAI et TANAKA, 1980; OCHIAI et al., 1980; ELIASSEN et VAHL, 1982 apud MUKHOPADHYAY et SINHA, 1986).

De variations annuelles du contenu de lipides chez Clupea harengus et d'autres poissons marins, peuvent être directement associées avec la disponibilité de nourriture (HARDY et MACKIE, 1969; HARDY et KEAY, 1972; HAYASHI et TAKAGI, 1978 apud HENDERSON et ALTAMAR, 1989). MAKAROVA (1975) a conclu que l'active consommation pour une partie des femelles du Perca fluviatilis sexuellement mûres, de poissons avec un contenu élevé de calories est fondamental pour maintenir le métabolisme énergétique et contructif pendant la période de la vitellogenèse.

\section{BIBLIOGRAPHIE}

ALLEN, K.R. The food and migration of the perch (Perca fluviatilis) in Windermere. J. Ani. Ecol, Oxford, 4:264-273, 1935. 
CECILIO, E.B.; AGOSTINHO, A. A. Biologia reprodutiva de Hyphophthalmus edentatus (Spix, 1829) (Osteichtyes, Siluriformes) no reservatório de Itaipu - PR. I. Estrutura dos testículos e escala de maturidade. Revista Unimar. Maringá, 13(2):195209, 1991.

CHAVES, P.T.C. Hidratação pré-ovulatória em peixes = um carácter de origem marinha? Revista Bras. Zool. Curitiba, 6(3):463-472, 1989.

DAWSON, A.S.; GRIMM, A.S. Quantitative seasonal changes in the protein, lipid and energy content of the carcass, ovaries and liver of adult female plaice, Pleuronectes platessa, L. J. Fish Biol. London, 16:493-504, 1980.

GARCIA, T.; PHILLIP, P. Desarrollo de los ovocitos en la tilapia Oreochromis aureus. Ver. Invest. Mar. Ciudad de la Habana, 7(2):63-70, 1986.

GUISANDE, C.; GLIWICZ, Z.M. Egg size and clutch size in two Daphnia species grown at different food level. J. Plankton Res. Oxon, 14(7):997-1007, 1992.

HENDERSON, R. J.; ALMATAR, S.M. Seasonal changes in the lipid composition of herring (Clupea harengus) in relation to gonad maturation. J. Mar. Biol. Assoc. U.K. Cambridge, 69:323-334, 1989.

HOUILlON, C. Sexualidade. São Paulo, Edgar Bücher, 1972, $171 \mathrm{p}$.

ISAAC-NAHUM, V.J.; VAZZOLER, A.E.A. de M.; ZANETTI-PRADO, E.M. Estudos sobre estrutura, ciclo de vida e comportamento de Sardinella brasiliensis (Steindachner, 1879), na área entre $22^{\circ} \mathrm{S} \mathrm{e}$ $28^{\circ} \mathrm{S}$, Brasil, 3. Morfologia e histologia de ovários e escala de maturidade. Bol. Inst. Oceanogr. São Paulo, 32(1):1-16, 1983.

MAKAROVA, N.P. Differences in the biological indicators of sexually mature and imature female perch Perca fluviatilis, from Uglich reservoir. J. Ichthyol. (Engl. Transl. Vopr. Ikthiol), Bethesda, 15(2):330-334, 1975.

MARGALEF, R. Ecología. Barcelona: Omega, 1977. $951 \mathrm{p}$.
MATSUURA, S.; MATSUYAMA, M.; OUCHI, Y.; HIDAKA, T. Maturity classification and group maturity of the red sea bream Pagrus major. II. Male maturity. Mar. Biol. (Berl.). Heidelberg, 96:169-172, 1987.

MOSILLE, O. I.W.; MAINOYA, J.R. Reproductive biology of the East African lung fish (Protopterus aethiopicus) in Mwanza Gulf, Lake Victoria. Afr. J. Ecol. Oxford, 26:149-162, 1988.

MUKHOPADHYAY, S.; SINHA, G.M. Seasonal variations in protein, lipid and carbohydrate contents of testes, liver and muscle in relation to spermatogenesis of an Indian freshwater major carp, Cirrhinus mrigala (Hamilton). Bol. Fisiol. Anim. (São Paulo ). 10:79-88, 1986.

RIMMER, M.A. Reproductive cycle of the fork-tailed catfish Arius graeffei Kner \& Steindachner (Pisces: Ariidae) from the Clarence river, New South Wales. Aust. J. Mar. Freschwater Res. East Melbourne, 36:23-32, 1985.

ROMAGOSA, E.; PAIVA, P. de; GODINHO, H.M.; STORFER, E.B. Desenvolvimento dos ovócitos de Piaractus mesopotamicus (Holmberg, 1887) (= Colossoma mitrei Berg, 1895) em condições de cultivo intensivo. Ciência e Cultura (São Paulo). 40(1):60-64, 1988.

RUIZ, C.; MARTINEZ, D.; MOSQUERA, G.; ABAD, M.; SANCHEZ, J.L. Seasonal variations in condition, reproductive activity and biochemical composition of the flat oyster, Ostrea edulis, from San Cibran (Galicia Spain). Mar. Biol. (Berl.). Heidelberg, 112(1):67-74, 1992.

WORSMANN, T.U.; MANDELLI, M.Q. Observações morfológicas sobre o processo de maturação do ovário de Sardinella brasiliensis. Revista Bras. Biol. Rio de Janeiro, 36(2):347-352, 1976.

ZANIBONI FILHO, E.; RESENDE, E.K. Anatomia de gônadas, escala de maturidade e tipo de desova do matrinxã, Brycon cephalus (Günther, 1869) (Teleostei: Characidae). Revista Bras. Biol. Rio de Janeiro, 48(4):833-844, 1988. 